\title{
LA-UR-20-26967
}

Approved for public release; distribution is unlimited.

Title: $\quad$ Salts in Hot Water: Developing a Scientific Basis for Supercritical Desalination and Strategic Metal Recovery

Author(s): $\quad$ Maerzke, Katie A.

Pratt, Lawrence R

Intended for: institutional computing proposal

Issued: 2020-09-04 
Disclaimer:

Los Alamos National Laboratory, an affirmative action/equal opportunity employer, is operated by Triad National Security, LLC for the National Nuclear Security Administration of U.S. Department of Energy under contract 89233218CNA000001. By approving this article, the publisher recognizes that the U.S. Government retains nonexclusive, royalty-free license to publish or reproduce the published form of this contribution, or to allow others to do so, for U.S. Government purposes. Los Alamos National Laboratory requests that the publisher identify this article as work performed under the auspices of the U.S. Department of Energy. Los Alamos National Laboratory strongly supports academic freedom and a researcher's right to publish; as an institution, however, the Laboratory does not endorse the viewpoint of a publication or guarantee its technical correctness. 


\section{Salts in Hot Water: Developing a Scientific Basis for Supercritical Desalination and Strategic Metal Recovery}

\section{Project Summary Information:}

\begin{tabular}{|r|l|l|l|l|}
\hline \multicolumn{5}{|c|}{ Summary Information } \\
\hline Project moniker & superdesal & Email & Z\# & Group \\
\hline Principal Investigator & Name & kmaerzke@lanl.gov & 292399 & T-1 \\
\cline { 2 - 5 } & Katie Maerzke & Email & Organization \\
\hline Co-investigators & Name & lpratt@tulane.edu & Tulane University \\
\cline { 2 - 6 } & Lawrence Pratt & \multicolumn{2}{l}{} \\
\hline Funding source/program & LDRD 20190057DR &
\end{tabular}

Abstract: Given the steady growth in world population and the ever-increasing fresh water demand, the only sustainable water supply option is desalination. Conceptually, desalination is very simple - just separate dissolved salts from water. While desalination research programs have existed since the 1960s and have resulted in a multitude of approaches, realizing affordable desalination has proven to be a challenge. Our goal is to meet this challenge using low cost heat sources to drive a liquid-discharge-free desalination process that can be co-mingled with the extraction of economically valuable co-products. High temperatures and pressures will be used to manipulate water's properties, such as the dielectric constant, and hence its ability to solvate ions. Selective precipitation/recovery of valuable metal co-products creates new revenue streams (i.e., cost offsets). We will develop the scientific understanding necessary to control salt precipitation from supercritical seawater, inland brines, and water commonly co-produced with oil and gas. Atomistic simulations will be used to understand at a molecular level the changes in hydrogen bonding and ion solvation that occur as we increase the temperature and/or pressure and change the density and ion concentration. Our simulation results will also aid in the interpretation of experimental data and guide the development of applied thermodynamic models for engineering design calculations.

Technical Description: Affordable desalination should include the use of less-expensive energy and comingle desalination with other profitable ventures. Specifically, if a desalination method could be driven with less expensive thermal energy sources and include the recovery of strategic metals as co-products that offset costs, it would be game changing. Our proposed method (LDRD 20190057DR) accomplishes this by selectively precipitating salts from supercritical water. This innovative method (a) exploits molecular properties of water under supercritical (SC) conditions, (b) recovers valuable metals (e.g., lithium and rare earth elements), and (c) can be powered by inexpensive energy sources (e.g., thermal energy). By controlling temperature and density, the properties of water can be manipulated. Water essentially acts as a dielectric to screen ionic charges, which is central to its role as a solvent. However, the dielectric constant degrades significantly above the critical point $\left(T_{\mathrm{C}}=647 \mathrm{~K}, P_{\mathrm{C}}=218 \mathrm{~atm},{ }_{\mathrm{C}}=0.322 \mathrm{~g} / \mathrm{cm}^{3}\right)$. Under SC conditions, dielectric properties are altered dramatically as the thermal energy associated with the higher temperature randomizes the water dipole orientations and disrupts the hydrogen bond network. Under lower density SC conditions, water's ability to solvate ions and ionic complexes is degraded and it becomes a surprisingly poor solvent for ions. ${ }^{1-4}$ Salt precipitation under SC conditions is well documented. For example, measurements of $\mathrm{NaCl}$ solubility in $\mathrm{SC}$ water ${ }^{5,6}$ show its solubility to be below the limit required for drinking water. Salt precipitation from $\mathrm{SC}$ water containing $3.5 \mathrm{wt} . \% \mathrm{NaCl}$ has also been demonstrated in the context of seawater desalination. ${ }^{7-10}$ Low salt solubility in water is also encountered in SC oxidation processes for destruction of organic wastes. ${ }^{11,12}$

However, we are not just interested in simple salts like $\mathrm{NaCl}$. Deep aquifer brines vary widely in composition. The same is true of many other potential water sources. For example, wastewaters from precious metal refining nominally have tens of ppm of $\mathrm{Au}, \mathrm{Pt}, \mathrm{Pd}$, and $\mathrm{Ag} .{ }^{13}$ Acid mine drainage is typically 
depleted in precious metals, but contains elevated concentrations of transition metals, such as $\mathrm{Cu}, \mathrm{Ni}$, or $\mathrm{Co}$ and tens to hundreds of ppb of the rare earths. ${ }^{14}$ Flow-back water associated with fracking is poorly documented but platinum group elements, ppm levels of Li, and tens of ppm of Sr have been reported. ${ }^{15}$ Thus, desalination co-mingled with metal co-production must be tunable to specific source waters. This requires a general predictive understanding of SC salt solubility.

The common view of dissolved salt is that water surrounds and electrostatically shields the individual ions. However, under SC conditions dipole orientations and the hydrogen bond network are disrupted. Thus, water can no longer shield charges and strongly bound ion pairs form. These ion pairs are held in solution by water clusters. Precisely how ion-ion association, cluster size distributions, and cluster-cluster interactions affect nucleation, growth, and precipitation of salt is not well understood in SC fluids. Atomistic simulations will provide molecular level understanding the changes in hydrogen bonding, ion speciation, and the degree of clustering (a precursor to precipitation) that occur as we increase the temperature and/or pressure, increase or decrease the density, and change the salt concentration and composition. Our simulation results will provide fundamental molecular-level insight as well as aid in the interpretation of experimental data and the development of applied models for engineering design calculations.

A combination of first principles (density functional theory, DFT) and classical simulations will be used to calculate dielectric constants and observe changes in hydrogen bonding, evaluate the accuracy of models in predicting phase equilibria, understand dynamical properties, the coordination of dissolved ions, ${ }^{16}$ the prevalence of ion pairing, and clustering (a precursor of salt precipitation). We will use a variety of techniques to accomplish these goals. Conventional molecular dynamics (MD) simulations are often an efficient choice of simulation method; however, under certain circumstances MD can run into time and/or length scale issues where sampling becomes problematic. For instance, simulating vapor-liquid phase equilibria and clustering in strongly associating fluids. In these cases, specialized Monte Carlo (MC) algorithms are the better choice. These algorithms include Gibbs Ensemble MC (GEMC) ${ }^{17,18}$ and Aggregation-Volume-Bias MC (AVBMC). ${ }^{19}$ First principles MC simulations are extremely expensive; however, with the use of more accurate machine-learned nested potentials the computational cost is less prohibitive. ${ }^{20}$ Finally, we will also perform electronic structure calculations to help parameterize and validate semi-empirical solubility models. ${ }^{21}$

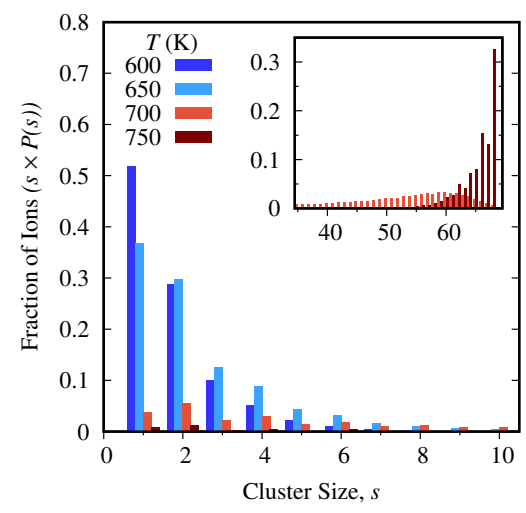

Figure 1: Analysis of the cluster size distributions of $\mathrm{NaCl}$ in water.
We will continue to build on our experience simulating aqueous electrolytes. Our earlier work on dynamic properties ${ }^{22}$ as well as an evaluation of many of the common classical force field for aqueous $\mathrm{NaCl}^{23}$ has given us a good foundation from which to proceed. We will use MD simulations in GROMACS to calculate potentials of mean force (PMF), or the relative free energy differences between solvated ions, contact pairs, and solvent-separated ion pairs. ${ }^{24}$ By using inexpensive force fields we will be able to explore a much wider range of state points, which will enable to see how the differences in relative free energy change as we change the temperature, density, ionic charge, and ionic radius. We will also study mixtures with more than one type of dissolved salt (using MD, MC, or both). Simulations of mixtures will allow us to probe how different ions interact with one another. For instance, the presence of other salts may either enhance or suppress cluster formation via "salting-out" or "salting-in" effects. ${ }^{25}$ This work will build on our understanding of clustering in aqueous $\mathrm{NaCl}$ solutions $\mathrm{s}^{23}$ (see Figure 1). Finally, we will explore more challenging counter-ions, e.g., common naturally-occurring carbonate and sulfate ligands. 
In addition to classical simulation, we will perform first principles simulations using CP2K. It is welldocumented in the literature that aqueous vapors and low-density supercritical fluids play a very important role in metal transport. Experimental data suggests that the solubility of copper in these high-temperature vapors in controlled by the formation of hydrated clusters of the form $\mathrm{CuCl}\left(\mathrm{H}_{2} \mathrm{O}\right)_{n}$, where the average number of water molecules in the cluster increases with increasing density. ${ }^{26}$ However, the nature of these clusters is difficult to probe experimentally. Moreover, there are some discrepancies between the experimental data and prior simulation work..$^{27}$ We have performed first principles simulations to explore these clusters in more detail. Through our initial simulations on high temperature aqueous $\mathrm{CuCl}$ vapors, we have learned that the molecular dynamics does indeed exhibit a sampling problem, especially for larger system sizes where almost all of the water molecules form a single large cluster (see Figure 2). Thus, MC is the appropriate technique for this type of simulation. We have also learned that using a more accurate "nested" potential for MC simulations increases the efficiency of these costly simulations. ${ }^{20,28}$ Our preliminary results for $\mathrm{CuCl}$ have greatly improved the agreement with experiment compared to earlier MD simulations due to our choice of technique as well as DFT functional. ${ }^{26,27,28}$ Our simulations help validate the experiments, as well as increase our understanding of what is happening on a molecular level under these conditions. We find a broad distribution of hydration numbers (that is, the number of water molecules in the $\mathrm{CuCl}\left(\mathrm{H}_{2} \mathrm{O}\right)_{n}$ cluster), especially at higher water densities. Moreover, these clusters are highly asymmetric, with $\mathrm{CuCl}$ primarily residing at the edge of the water cluster. In FY21 we will build on this work by extending our simulations to additional temperatures, salts, and counterions. We will initially focus on $\mathrm{AgCl}$ and $\mathrm{AuCl}$, which will allow to explore changing trends in hydration as we move down the periodic table. Additionally, we plan to study $\mathrm{LaCl}_{3}, \mathrm{NdCl}_{3}$, and $\mathrm{ErCl}_{3}$, a light, intermediate, and heavy lanthanide, respectively.

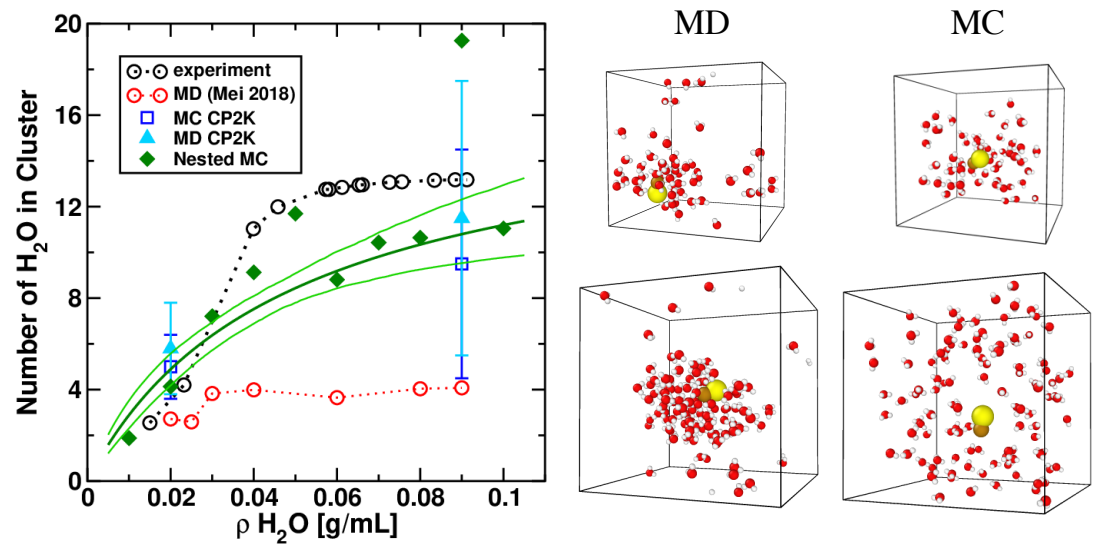

Figure 2: Preliminary results for aqueous high temperature $\mathrm{CuCl}$ vapors. Plot comparing the average hydration number against the experimental data (left) as well as simulation snapshots from MD and MC simulations. Top snapshots show simulations with $55 \mathrm{H}_{2} \mathrm{O}$ molecules, bottom snapshots show simulations with $110 \mathrm{H}_{2} \mathrm{O}$ molecules. The 110 water molecule MD simulation indicates a sampling problem, which is not present in the preliminary $\mathrm{MC}$ simulation.

We are requesting an additional year of computer time in order to complete and extend the work we began under our earlier allocation, w19_superdesal. This work is in support of an LDRD-DR project (20190057DR).

Project Plan: Since this LDRD-DR project ends with FY21, we plan to focus our efforts on completing the simulations by August 2021 to allow enough time to analyze and write up results before our project is over. We are requesting computer time to complete work that we have already begun, and thus have all of the necessary codes compiled, submission scripts in place, and a reasonable workflow. We are on track to use - or even slightly exceed - our allocation for 2020. Given all of this, we do not see any major risks associated with this project.

Team Qualifications and Prior Experience: The PI, Katie Maerzke, has more than 15 years of experience in HPC computing. She spent her graduate career as a developer and user of MCCCS-MN and debugged the first parallel version of that code. Since graduate school, she has run GROMACS simulations on HPC resources at Notre Dame and LANL, along with Gaussian calculations. She is the PI for LANL IC project w19_superdesal, where she has used the bulk of the allocation $(>75 \%)$ to run CP2K 
simulations for aqueous $\mathrm{CuCl}$ vapors. She has more than 15 publications that have relied on HPC resources. Co-PI Lawrence Pratt is the Herman and George R. Brown Chair at Tulane University. Dr. Pratt's contributions to thermodynamics and liquid state theory have earned him an international reputation for scientific excellence. Postdocs Tae Jun Yoon and Lara Patel have compiled LAMMPS and GROMACS, respectively, and have numerous publications detailing the results of MD simulations. Postdoc Chris Alcorn has used Gaussian16 for electronic structure calculations, which have contributed to several publications.

HPC and Parallel Computing Details: Classical Monte Carlo simulations will be performed using the

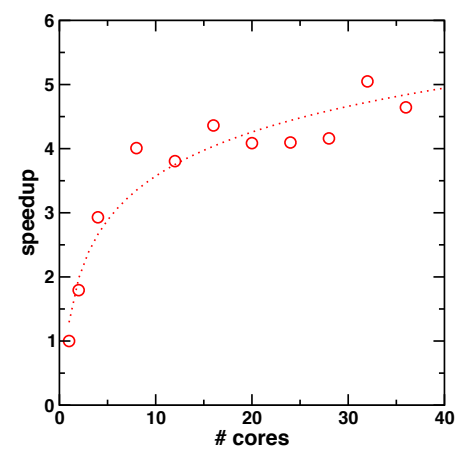

Figure 3: Parallel speedup for MCCCS-MN on Grizzly. MCCCS-MN software package developed by the Siepmann group at the University of Minnesota. ${ }^{29}$ MCCCS-MN supports all the common statistical ensembles. A variety of advanced, state-of-art sampling algorithms are implemented in the software, including AVBMC algorithms to sample the spatial distribution of associating fluids. MCCCS-MN is written in Fortran 90 and uses hybrid MPI/OpenMP for parallel execution. It has no other reliance on external libraries, which is a design decision to ensure computational-platform neutrality. MCCCS-MN does not scale to a very large number of cores (see Figure 3); however, multiple simulations can be launched from a single submission script. Four eight-core jobs can be run with a single submission to Grizzly without any file I/O issues. In this way, we can easily run multiple replicas of the same system (for better statistics) or screen a larger number of state points.

The $\mathrm{CP} 2 \mathrm{~K}$ code ${ }^{30}$ is based on Gaussian plane wave approach and uses various original state of the art algorithms to reduce time spent on SCF interactions, e.g., the auxiliary density matrix method for Hartree-Fock exchange calculations and the orbital transformation based iterative refinement for direct energy functional minimization under orthogonality constraints. In order to compile CP2K written in Fortran 90, several libraries are needed: LAPACK and SCALAPACK for linear algebra, the IBM-provided Engineering and Scientific Subroutine Library (ESSL) for general calculations, and the FFTW3 library for Fourier Fast transforms. For a wide variety of XC functionals, the LIBINT and LIBXC libraries are needed. CP2K uses the DBCSR library for efficient sparse matrix multiplication. The PI has compiled and run $\mathrm{CP} 2 \mathrm{~K}$ on Snow and Grizzly. For the relatively small number of atoms in the simulations we propose, $\mathrm{CP} 2 \mathrm{~K}$ runs efficiently on 4-8 nodes (see Figure 4). Note that due to the computational expense, we have not tested

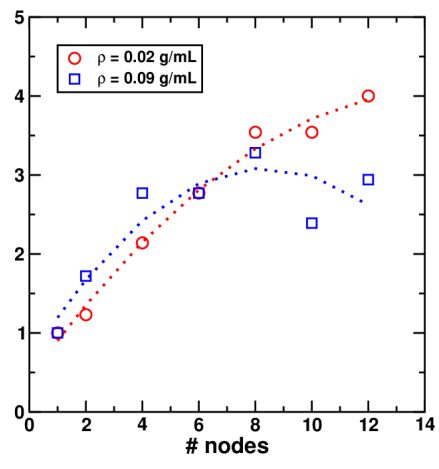

Figure 4: Scaling behavior of $\mathrm{MC}$ simulations with $\mathrm{CP} 2 \mathrm{~K}$ for $55 \mathrm{H}_{2} \mathrm{O}+1 \mathrm{CuCl}$ at low densities on Grizzly. $\mathrm{CP} 2 \mathrm{~K}$ on fewer than 1 node/36 cores. CP2K does not scale as well with simulation box size; $5 \mathrm{MC}$ steps on 1 node take approximately 12 wallclock hours at a density of $0.02 \mathrm{~g} / \mathrm{mL}$ and only 4.75 hours at a density of $0.09 \mathrm{~g} / \mathrm{mL}$. For this reason the vapor phase simulations we propose require more CPU-hrs than simulations in a condensed phase. Additional information on CP2K may be found on the website. ${ }^{30}$

GROMACS and LAMMPS are widely used, freely available, efficient and highly parallel molecular dynamics codes. They both scale to hundreds of cores, though such a large number of cores is not necessary for the modest size simulations we propose..$^{31-35}$

Gaussian $16^{36}$ is a well-established state-of-the-art electronic structure calculation code. It scales well across shared-memory processors, but the performance suffers when communication across nodes is required. For the relatively small-scale calculations we propose, a single core will be sufficient. 


\section{$\underline{\text { Resource Requirements: }}$}

1) 500 classical Monte Carlo simulations on 1 node of Badger. These simulations will take an average of 200 wallclock hours each. 1 job submission to Badger will run 4 simulations; hence we'll need to run 125 simulations.

125 jobs $\times 200 \mathrm{hrs} / \mathrm{job}=25,000 \mathrm{hrs}$ on 1 node $(=25,000 \mathrm{CPU}-\mathrm{hrs})$

Data storage needs will not be high for these MC simulations. We estimate

1 GB per simulation, for a total of 500 GB.

2) Classical molecular dynamics simulations with GROMACS and/or LAMMPS on 1-10 nodes of Badger (36 cores/node). Time estimates were arrived at by scaling up previous simulations on Wolf (16 cores/node). A 5 ns run with 10,000 atoms took 4.5 hours on 1 node of Wolf and a 1 ns run with 100,000 atoms took 7.5 hours on 1 node of Wolf.

a) 10,000 atom simulations: 200 scoping runs of $20 \mathrm{~ns}$ and 50 high resolution runs of $200 \mathrm{~ns}$ for a total of $14,000 \mathrm{~ns}$ of MD simulation.

$(4.5 \mathrm{hrs} / 5 \mathrm{~ns}) \times 20,000 \mathrm{~ns} \times(16 / 36$ cores $)=5,600 \mathrm{hrs}$ on 36 cores or 1 node

b) 100,000 atoms simulations: 100 runs of $20 \mathrm{~ns}$ for a total of $2000 \mathrm{~ns}$ of MD simulation $(7.5 \mathrm{hrs} / 1 \mathrm{~ns}) \times 2000 \mathrm{~ns} \times(16 / 36$ cores $)=6600 \mathrm{hrs}$ on 36 cores

c) Potential of Mean Force (PMF) calculations. Each PMF will be calculated from 2.2-10.0 $\AA$ at a spacing of $0.2 \AA$ for a total of 40 simulations. Each simulation will have 10,000 atoms and run for $10 \mathrm{~ns}$, for a total of $400 \mathrm{~ns}$ of simulation time per PMF. We estimate that we'll calculate 20 PMFs with 4 independent replicas each for accurate statistics.

$(4.5 \mathrm{hrs} / 5 \mathrm{~ns}) \times(400 \mathrm{~ns} / \mathrm{run}) \times 80$ runs $\times(16 / 36$ cores $)=12,800 \mathrm{hrs}$ on 36 cores .

Total request for classical MD simulations: 25,000 CPU-hrs. Data storage needs will be higher for these MD runs. We estimate we will need 9 TB.

3) First principles simulations with CP2K. Through our work on IC project w19_superdesal, we have learned which technique (MC) and how many cores to use to efficiently perform $\mathrm{CP} \overline{\mathrm{K}}$ simulations of low density phases. For the relatively small number of atoms in the simulations we propose (165-350 atoms) $\mathrm{CP} 2 \mathrm{~K}$ runs efficiently on 8 nodes of Grizzly. We estimate that the average state point requires approximately 25,000 CPU-hrs (3125 wallclock hours). We propose performing simulations over a range of 10 densities for 5 different metals (Au, Ag, La, Nd, Er) at two temperatures, for a total of 100 state points. For more accurate statistics, we will perform at least 2 independent simulations for each state point, for a total of 200 simulations.

200 simulations $\mathrm{x} 25,000$ CPU-hrs $=5,000,000$ CPU-hrs.

Due to the small system sizes, data storage needs will not be very high for each state point, at most 2 GB. This will require $400 \mathrm{~GB}$ total.

The resources required for Gaussian calculations and training the machine-learned nested potential for use with $\mathrm{CP} 2 \mathrm{~K}$ are negligible; thus, we have not specifically requested CPU-hrs for these calculations.

\section{Conclusion:}

Therefore, we propose a total one-year allocation of 5,050,000 CPU-hrs on Badger and Grizzly: 25,000 for (1) $+25,000$ for (2) +5,000,000 for (3). This should be divided into 50,000 CPU-hrs on Badger for (1) and (2) and 5,000,000 CPU-hrs on Grizzly for (3). Total data storage needs are 10 TB in Lustre scratch space and Campaign Storage space. Many of the large trajectory files may be deleted after publication of the resulting paper; hence, we do not need as much archival storage space. We ask for $5 \mathrm{~TB}$ Archival Space. 


\section{REFERENCES:}

1. K. S. Pitzer, "Dielectric Constant of Water at Very High Temperature and Pressure" PNAS USA Vol. 80: 4575-4576 (1983).

2. H. Weingartner and E. U. Franck, "Supercritical Water as a Solvent" Angewandte Chemie Internat. Ed. 44: 2672-2692 (2005) ; also see: I. Pioro and S. Mokry, "Thermophysical Properties at Critical and Supercritical Conditions" Chapter 22 in Heat Transfer - Theoretical Analysis, Experimental Investigations and Industrial Systems A. Belmiloudi, Editor, InTech (2011).

3. A. A. Galkin and V. V. Lunin, "Subcritical and Supercritical Water: A Universal Medium for Chemical Reactions" Russian Chemical Reviews 74(1): 21-35 (2005).

4. J. V. Walther, "Mineral Solubilities in Supercritical $\mathrm{H}_{2} \mathrm{O}$ Solutions" Pure \& Applied Chemistry 58 (12): 1585-1598 (1986).

5. S. T. Cui and J. G. Harris, "Solubility of Sodium Chloride in Supercritical Water: A Molecular Dynamics Study" Journal of Physical Chemistry 99: 2900 (1995).

6. F. Armellini, F. J. Tester, and G. T. Hong, "Precipitation of Sodium Chloride and Sodium Sulfate In Water from Sub- to Supercritical Conditions: 150 to $550{ }^{\circ} \mathrm{C}, 100$ to 300 bar" Journal of Supercritical Fluids 7: 147 (1994).

7. S.O. Odu, A. G. J. van der Ham, S. Metz, and S. R. A. Kersten, "Design of a Process for Supercritical Water Desalination with Zero Liquid Discharge" Industrial \& Engineering Chemistry Research 54: 5527-5535 (2015).

8. I. Leusbrock, S. J. Metz, G. Rexwinkel, and G. F. Versteeg, "Quantitative Approaches for the Description of Solubilities of Inorganic Compounds in Near-critical and Supercritical Water" Journal of Supercritical Fluids 47: 117-127 (2008).

9. I. Leusbrock, S. J. Metz, G. Rexwinkel, and G. F. Versteeg, "Solubility of 1:1 Alkali Nitrates and Chlorides in Near-Critical and Supercritical Water" Journal of Chemical and Engineering Data 54: $3215-$ 3223 (2009).

10. M. Schubert, J. W. Regler, and F. Vogel, "Continuous Salt Precipitation and Separation from Supercritical Water. Part 1: Type 1 Salts" Journal of Supercritical Fluids 52: 99-112 (2010); "Continuous Salt Precipitation and Separation from Supecritical Water. Part 2. Type 2 Salts and Mixtures of Two Salts" Journal of Supercritical Fluids 52: 113-124 (2010); "Continuous Salt Precipitation and Separation from Supercritical Water. Part 3: Interesting Effects in Processing Type 2 Salt Mixtures" Journal of Supercritical Fluids 61: 44-54 (2012).

11. F .J. Armellini and J. W. Tester, "Solubility of Sodium Chloride and Sulfate in Sub- and Supercritical Water Vapor from 450-550 ${ }^{\circ} \mathrm{C}$ and 100-250 Bar" Fluid Phase Equilibria 84: 123-142 (1993).

12. M. Hodes, P. A. Marrone, G. T. Hong, K. A. Smith, and J. W. Tester, "Salt Precipitation and Scale Control in Supercritical Water Oxidation-Part A: Fundamentals and Research" Journal of Supercritical Fluids 29: 265 (2004).

13. H. Umeda, A. Sasaki, K. Takahashi, K. Haga, Y. Takasaki, and A. Shibayama, "Recovery and Concentration of Precious Metals from Strong Acidic Wastewater" Mater. Trans. 52: 1462-1470 (2011).

14. E. Ferreira da Silva, I. Bobos, J. X. Matos, C. Patinha, A. P. Reis, and E. C. Fonseca, "Mineralogy and Geochemistry of Trace Metals and REE in Volcanic Massive Sulfide Host Rocks, Stream Sediments, Stream Waters and Acid Mine Drainage from the Lousal Mine Area (Iberian Pyrite Belt, Portugal)" Appl. Geochemistry. 24: 383-401 (2009). 
15. Y. Lester, I. Errer, E. M. Thurman, K. A. Sitterley, J. A. Korak, G. Aiken, and K. G. Linden, "Characterization of Hydraulic Fracturing Flowback Water in Colorado: Implications for Water Treatment” Sci. Total Environ. 512-513: 637-644 (2015).

16. K. A. Maerzke, G. S. Goff, W. H. Runde, W. F. Schneider, and E. J. Maginn, "Structure and Dynamics of Uranyl and Plutonyl Ions in Ionic Liquid/Water Mixtures via Molecular Dynamics Simulation" J. Phys. Chem. B 117: 10852 (2013); K. A. Maerzke, G. S. Goff, W. H. Runde, W. F. Schneider, and E. J. Maginn, "Molecular Dynamics Simulations of Uranyl and Plutonyl Cations in a Task-Specific Ionic Liquid" J. Phys. Chem. B, in preparation.

17. A. Z. Panagiotopoulos, "Direct Determination of Phase Coexistence Properties of Fluids by Monte Carlo Simulation in a New Ensemble," Mol. Phys. 61: 813-826 (1987).

18. A. Z. Panagiotopoulos, N. Quirke, M. Stapleton, and D. J. Tildesley, "Phase Equilibria by Simulation in the Gibbs Ensemble - Alternative Derivation, Generalization and Application to Mixture and Membrane Equilibria," Mol. Phys. 63: 527-545 (1988).

19. B. Chen and J. I. Siepmann, "A Novel Monte Carlo Algorithm for Simulating Strongly Associating Fluids: Applications to Water, Hydrogen Fluoride, and Acetic Acid" J. Phys. Chem. B 104: 8725 (2000).

20. R. B. Jadrich and J. A. Leiding, "Accelerating Ab Initio Simulation via Nested Monte Carlo and Machine Learned Reference Potentials," J. Phys. Chem. B. 124: 5488-5497 (2020).

21. K. A. Velizhanin, C. D. Alcorn, A. A. Migdisov, and R. P. Currier, "Rigorous analysis of non-ideal solubility of sodium and copper chlorides in steam using Pitzer-Pabalan models," Fluid Phase Equilib. 522: 112731 (2020).

22. T. J. Yoon, L. A. Patel, M. J. Vigil, K. A. Maerzke, A. T. Findikoglu, and R. P. Currier, "Electrical conductivity, ion pairing, and ion self-diffusion in aqueous $\mathrm{NaCl}$ solutions at elevated temperatures and pressures," J. Chem. Phys. 151: 224504 (2019).

23. L. A. Patel, T. J. Yoon, R. P. Currier, and K. A. Maerzke, "NaCl Aggregation in Supercritical Water: Comparison of Classical Force Fields," in preparation.

24. G. M. Torrie and J. P. Valleau, "Nonphysical Sampling Distributions in Monte Carlo Free-Energy Estimation: Umbrella Sampling” J. Comp. Phys. 23: 187 (1977).

25. H. J. Reimer, F. Vogel, and M. Steele-MacInnis, "Speciation and Structural Properties of Hydrothermal Solutions of Sodium and Potassium Sulfate Studied by Molecular Dynamics Simulations" Chem. Phys. Chem. 17: 1446 (2016).

26. A. A. Migdisov, A. Y. Bychkov, A. E. Williams-Jones, and V. J. van Hinsberg, "A Predictive Model for the Transport of Copper by HCl-Bearing Water Vapour in Ore-Forming Magmatic-Hydrothermal

Systems: Implications for Copper Porphyry Ore Formation," Geochimica et Cosmochimica Acta 129: 33 53 (2014).

27. Y. Mei, W. Liu, A. A. Migdisov, J. Brugger, and A. E. Williams-Jones, "CuCl Complexation in the Vapor Phase: Insights from Ab Initio Molecular Dynamics Simulations," Geofluids 2018: 4279124 (2018).

28. K. A. Maerzke, R. B. Jadrich, T. J. Yoon, J. A. Leiding, and R. P. Currier, "First Principles Simulations of $\mathrm{CuCl}$ in High Temperature Water Vapor," in preparation.

29. J. I. Siepmann, M. G. Martin, B. Chen, C. D. Wick, J. M. Stubbs, J. J. Potoff, B. L. Eggimann, M. J. McGrath, X. S. Zhao, K. E. Anderson, J. L. Rafferty, N. Rai, K. A. Maerzke, S. J. Keasler, P. Bai, E. O. Fetisov, M. S. Shah, Q. P. Chen, R. F. DeJaco, J. L. Chen, X. Bai. Monte Carlo for Complex Chemical Chemical Systems - Minnesota, Version 18.1, Minneapolis, MN 2018.

http://siepmann.chem.umn.edu/software 
30. https://www.cp2k.org/

31. S. Pronk, S. Pall, R. Schulz, P. Larsson, P. Bjelkmar, R. Apostolov, M. R. Shirts, J. C. Smith, P. M. Kasson, D. van der Spoel, B. Hess, and E. Lindahl, "GROMACS 4.5: A High-Throughput and Highly Parallel Open Source Molecular Simulation Toolkit” Bioinfomatics 29: 845 (2013).

32. M. J. Abraham, T. Murtola, R. Schulz, S. Pall, J. C. Smith, B. Hess, and E. Lindahl, "GROMACS: High Performance Molecular Simulations Through Multi-Level Parallelism from Laptops to Supercomputers" SoftwareX, 1-2: 19 (2015).

33. http://www.gromacs.org/

34. S. Plimpton, "Fast Parallel Algorithms for Short-Range Molecular Dynamics", J. Comp. Phys., 117: 1-19 (1995).

35. http://lammps.sandia.gov

36. Gaussian 16, Revision C.01, M. J. Frisch, G. W. Trucks, H. B. Schlegel, G. E. Scuseria, M. A. Robb, J. R. Cheeseman, G. Scalmani, V. Barone, G. A. Petersson, H. Nakatsuji, X. Li, M. Caricato, A. V. Marenich, J. Bloino, B. G. Janesko, R. Gomperts, B. Mennucci, H. P. Hratchian, J. V. Ortiz, A. F. Izmaylov, J. L. Sonnenberg, D. Williams-Young, F. Ding, F. Lipparini, F. Egidi, J. Goings, B. Peng, A. Petrone, T. Henderson, D. Ranasinghe, V. G. Zakrzewski, J. Gao, N. Rega, G. Zheng, W. Liang, M. Hada, M. Ehara, K. Toyota, R. Fukuda, J. Hasegawa, M. Ishida, T. Nakajima, Y. Honda, O. Kitao, H. Nakai, T. Vreven, K. Throssell, J. A. Montgomery, Jr., J. E. Peralta, F. Ogliaro, M. J. Bearpark, J. J. Heyd, E. N. Brothers, K. N. Kudin, V. N. Staroverov, T. A. Keith, R. Kobayashi, J. Normand, K. Raghavachari, A. P. Rendell, J. C. Burant, S. S. Iyengar, J. Tomasi, M. Cossi, J. M. Millam, M. Klene, C. Adamo, R. Cammi, J. W. Ochterski, R. L. Martin, K. Morokuma, O. Farkas, J. B. Foresman, and D. J. Fox, Gaussian, Inc., Wallingford CT, 2016. 